\title{
Improvement of System to Fold T-shirt in the State of Hanging
}

\author{
Takumi Saruhashi ${ }^{\mathrm{a}}$, Ryunosuke Miyamoto ${ }^{\mathrm{b}}$, Shenglin $\mathrm{Mu}^{\mathrm{c}}$, Takaaki Akimoto ${ }^{\mathrm{a}}$, Yuhki Kitazono ${ }^{\mathrm{a}}$ \\ ${ }^{a}$ National Institute of Technology, Kitakyushu College,5-20-1 Shii, Kokuraminami-ku, Kitakyushu-city, \\ Fukuoka 802-0985, Japan \\ bMolex Japan Co., Ltd., 3949-8 Kawanishi-cho, Kanoya-city, Kagoshima 893-0032, Japan \\ ${ }^{\mathrm{c}}$ National Institute of Technology, Hiroshima College, 4272-1, Higashino, Osakikamijima-cho, Toyota-gun, \\ Hiroshima 725-0231, Japan
}

*Corresponding Author:kitazono@kct.ac.jp

\begin{abstract}
In this paper, we describe the T-shirt automatic fold system that we developed. This system, T-shirt on the hanger rack is automatically taken out whole the hanger, it will detect whether the laundry is dry or not. After that, the laundry is taken out from the hanger, and fold. After, the laundry that has been folded, it will be put away, and the hanger will be recovered. With this system, it will be able to alleviate some of the burden of housework.
\end{abstract}

Keywords: folding the laundry, folding system, robot, housework.

\section{Introduction}

Housework is one of tough jobs. Not only that, we must do it every day. We can't live comfortably without doing housework. In the case of a single life, you have to do that as well as your work. On the other hand, if you have a large family, amount of housework become enormous. There are a great number of household tasks such as cleaning the room, washing the dishes, and doing the washing ${ }^{(1,2)}$. Previously, housework has been done manually. However, as a result of appearance of Dishwasher, Vacuum cleaner, and Washing machine, we can conveniently save trouble. Nevertheless, the machine which folds the laundry automatically has not prevailed among the ordinary homes. Even today, folding the laundry is a manual labor.

There are several items to fold the $\mathrm{T}$-shirt ${ }^{(3)}$. For instance, Quick-Press sold by Daisaku Shoji Ltd. is item which make folding T-shirt easier. Although folding T-shirt became easier, it is manual procedure all the same. The folding machine which works automatically exist, however,

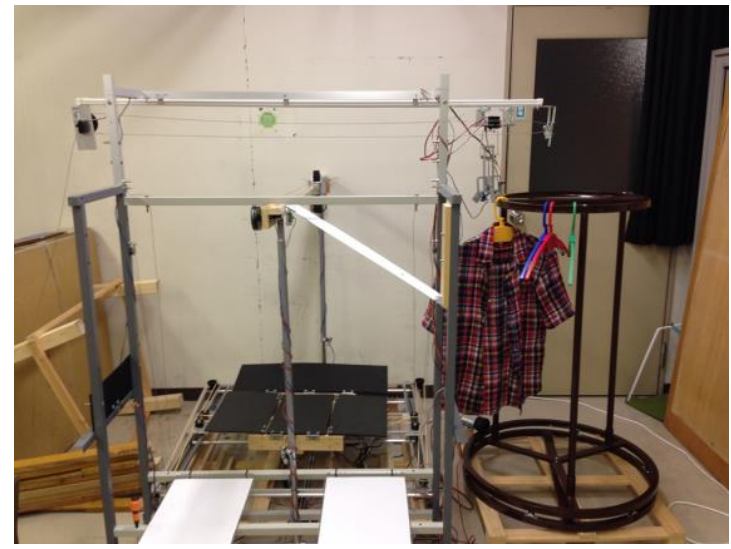

Fig. 1. Overall view of the system.

it is not automatic completely because it needs to put a T-shirt on it manually. There are two machines with function to fold T-shirt. However, one of them needs specific hanger which is expensive and complicated as many as the number of T-shirt. Another can fold a T-shirt hung on a hanger. However, owing to its mechanism to fold, some T-shirt can't be folded by this machine. Besides, these machines does not detect whether T-shirt is dry or not. Consequently, they needs some help by human to start folding.

So, we developed a system which folds the laundry in automatically ${ }^{(4)}$. Furthermore, it can detect whether the T-shirt is dry or not, while the T-shirt is hung on a hanger. The system can fold T-shirt precisely because it places the T-shirt on the table when it folds the T-shirt. All things that user have to do is only hanging washed T-shirt on a hanger. However, there was a limited number to fold the laundry in succession in once driving this system.

So, we improved the system that it can be folded much laundry in succession. 


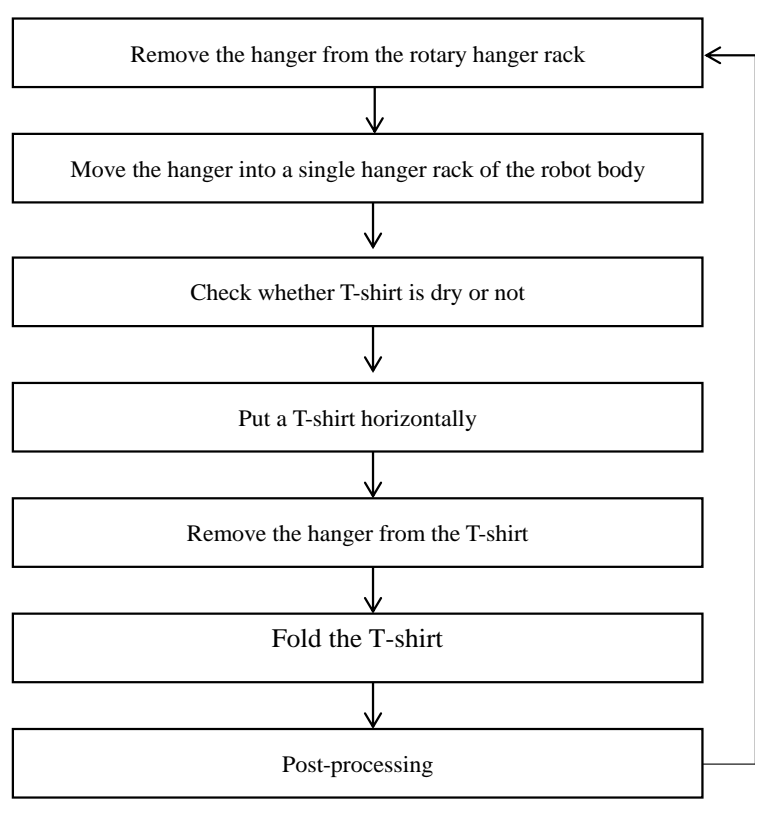

(a) Flowchart

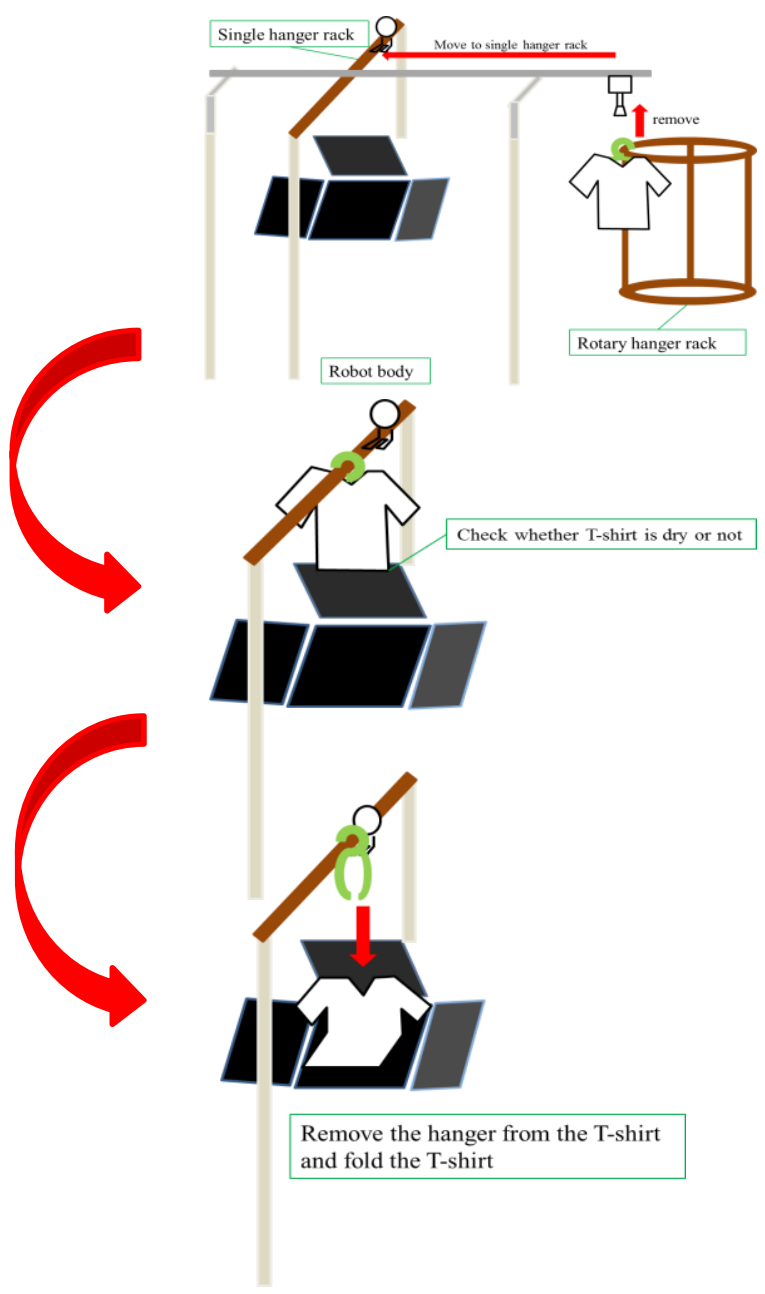

(b) Explanatory drawing

Fig. 2. System operation procedure.

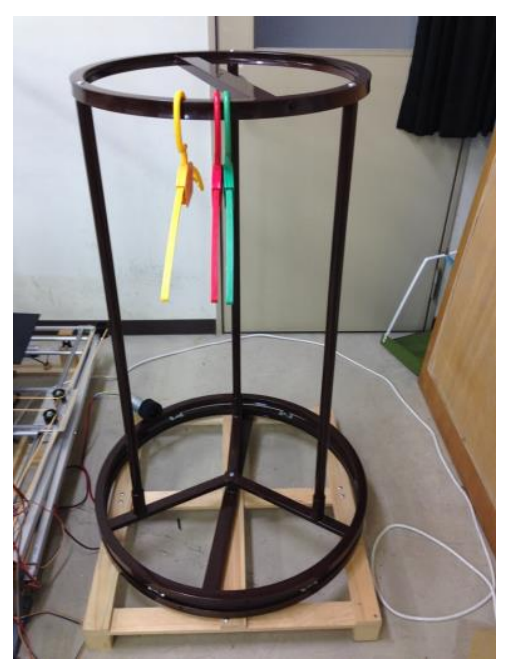

Fig. 3. Rotary hanger rack.

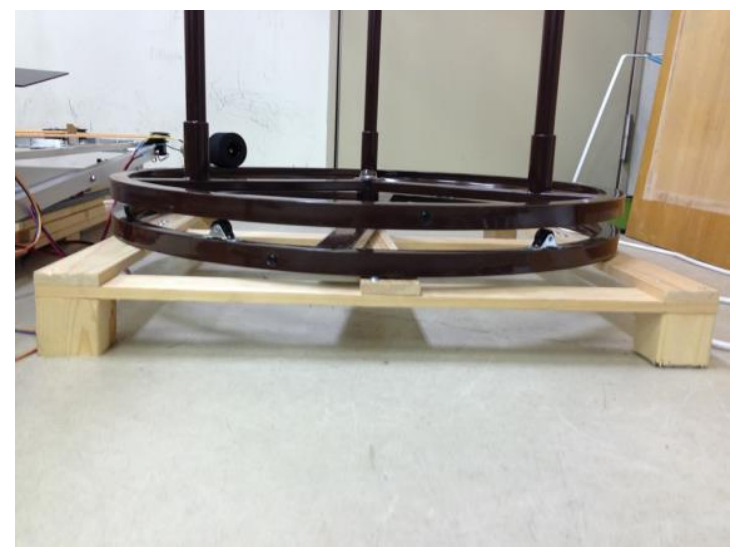

Fig. 4. Changed the height.

\section{System to Fold T-shirt Automatically}

\subsection{System summary}

The overall view of the fabricated system is shown in Fig.1. Rotary hanger rack of the right part is a place to put a T-shirt. A plurality of T-shirts that have been put to here is folded automatically by a robot body of the left part.

Briefly explaining the operation procedure of the system, first, take out a T-shirt along with hanger from rotary hanger rack. Next, system moves the hanger to the robot body, and checks by wet sensor whether it is dry or not. If it is dry, it is fold by the robot. It is shown in Fig.2 these operations.

\subsection{Removal of the hanger}

To fold in automatic a plurality of T-shirts, there is a need for a place where it can be to put of T-shirts. In this study, we use a rotary hanger rack as shown in Fig. 3 as a 


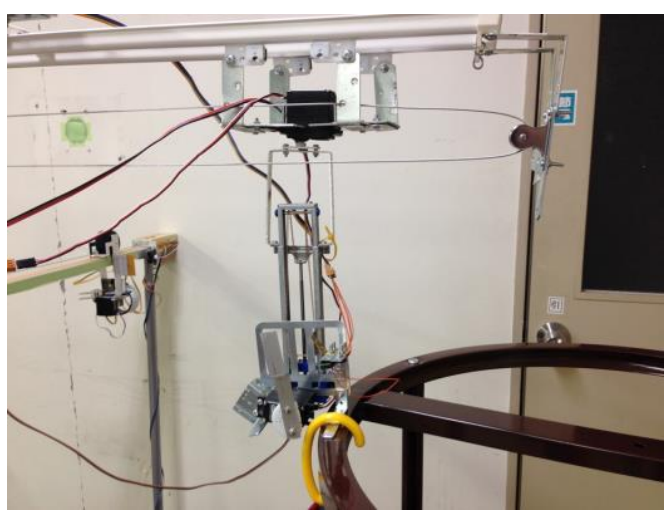

Fig. 5. Lift.

place to keep over a plurality of T-shirts. This time, we improved the mechanism for taking out the hanger from there. We have changed the height of the rotary hanger rack to low as shown the Fig.4.

Then, Lift carry hanger took to the hanger rack. Lift shown in Fig.5 is adapted to carry the hanger from the rotary hanger rack to single hanger rack which is robot body. As shown in Fig.6 the lift is attached two servo motors, one DC motor, and the distance measuring sensor, even more lift is attached to the curtain runner for curtain rail.

A rotary hanger rack is rotated to move hanger on the arm portion of the lift, and the distance measuring sensor which on the arm detects the top of the hanger hook. When distance measuring sensor detects the top of the hanger hook, the rotation of the rotary hanger rack is stop. And the servo motor which put on the arm of the lift is actuated for the hanger to not come off from the lift. When the servo motor is actuated, the arm which is attached to the servo motor is closed.

After that, the arm of the lift lifting a hanger by the DC motor. Then, servo motor which is attached one end of the curtain rail turn the pulley to move the curtain runners by winding the wire to move the hanger as shown in Fig.7. When the hanger moved to the midpoint of the single hanger rack and rotary hanger rack, the rotation of pulley is stop. The servo motor mounted on lift top is rotated 180 degrees to rotate the hanger in a direction to put a hanger to the single hanger rack. Then, the pulley rotates again to move the hanger to above the single hanger rack as shown in Fig.8.

After that, as shown in Fig.9, lowers the lift, and the servo motor arm is opened. By doing so, the hanger is falling by the weight of own from the lift to a single hanger rack. Then pulley is rotated in the reverse direction, and the servo motor attached to the lift top is rotated 180 degrees reverse. Then lift returns to its initial position and

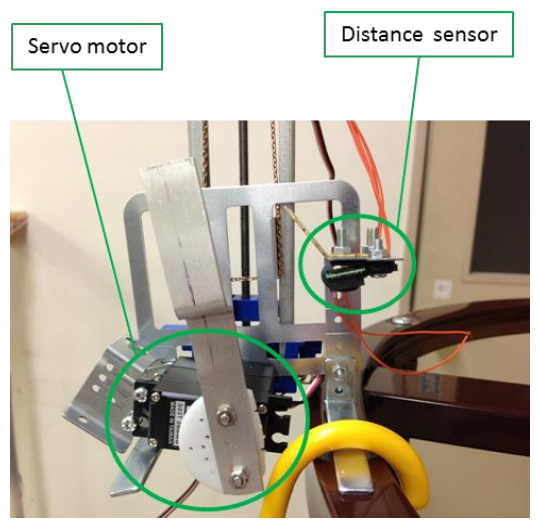

(a) One servo motor and sensor.

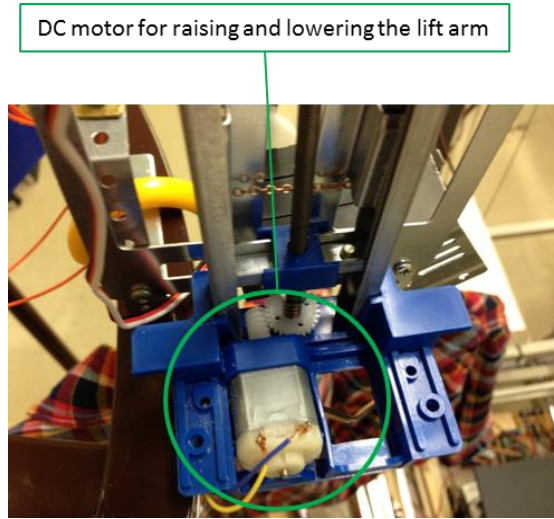

(b) One DC motor

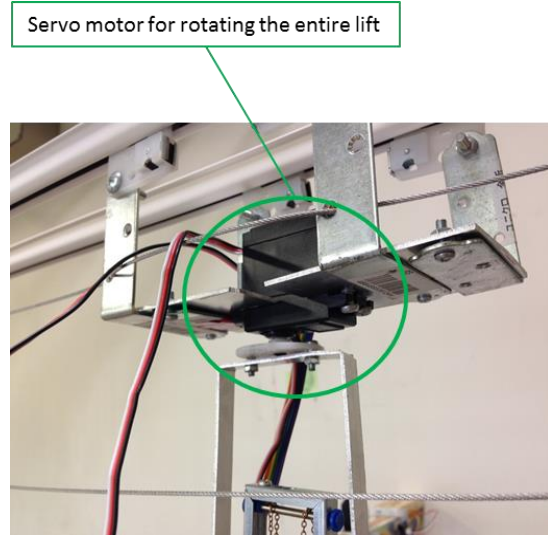

(c) The another servo motor and curtain rail

Fig. 6. Those was connected to a lift

wait for the next hanger.

This mechanism has become possible to move a many of hangers from the rotary hanger rack to a single hanger rack. 


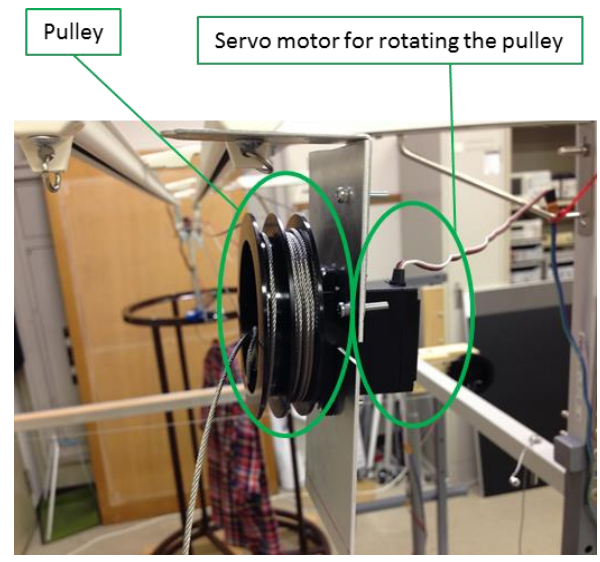

Fig. 7. Pulleys and servo motor.

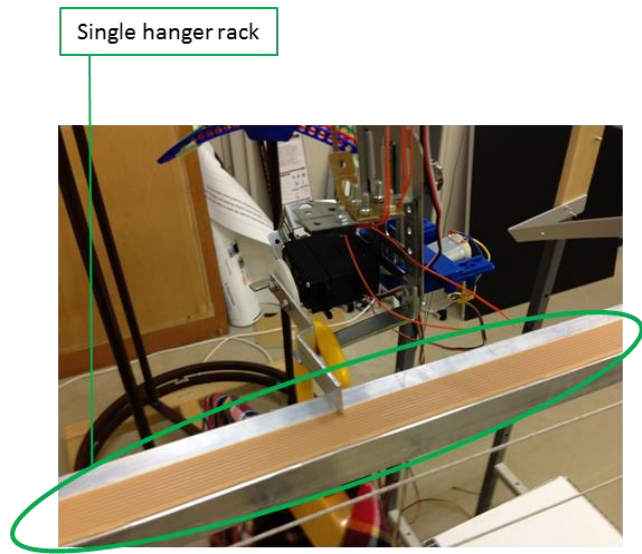

Fig. 8. Move the hanger to above the single hanger rack

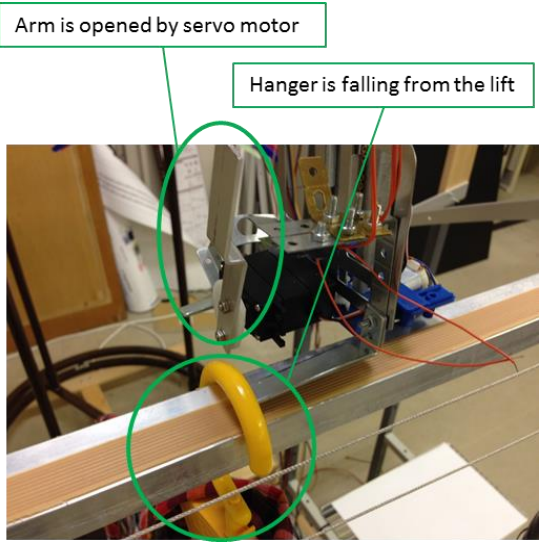

Fig. 9. Servo motor arm open

\subsection{Movement on a single hanger rack}

T-shirts that have been moved to on a single hanger rack is moved by the hanger mobile devices. To ensure the hanger handle by closing the hanger of the handle in the arm of the servo motor. Then, it moved to a predetermined position.

\subsection{Folding Instrument}

The folding instrument folds the T-shirt placed on it. The folding instrument consists of three boards. Folding these boards in order, it can fold T-shirt. Using this instrument, the system folds T-shirt.

\subsection{H-bot $^{(5)}$}

The H-bot is a system that operates the folding instrument shown in previous section. It moves from front to back and side to side. It has two motors at opposite side of corners. This system moves the central red stage in four directions with combinations of the rotation of these motors. The folding instrument is placed on the stage. When the two motors rotate in the same direction, the stage moves left or right. When the two motors rotate in the different direction, the stage moves front or back. The direction of movement is due to the direction of rotation.

\subsection{Lifting Mechanism}

This lifting mechanism lifts the folding instrument on the H-bot. It is called X-LINK LIFT. Thus, this mechanism moves up and down the system to fold a T-shirt.

\subsection{Wet Sensor ${ }^{(6)}$}

Resistance value of an object changes owing to the amount of water in the object. This nature of resistance can be used as a measure to detect whether the object is dry or not. In this system, the wet sensor checks the dryness of the T-shirt, measuring the resistance of the T-shirt.

\section{Manufacture}

We manufactured mechanism for taking out the hanger from the rotary hanger rack as shown in Fig.10. It has a mechanism for carrying the hanger: vertical movement, lateral movement, and rotation movement.

We're using a DC motor to vertical movement of the hanger. Lift the hanger by the lift arm up and down by a DC motor.

We have been using a wire, pulley, and the servo motor to lateral movement. It is passed through the wire to the part that connects the lift and curtain rail runner, and the wire is wrapped around pulley. By Pulley is rotated by a servo motor, wire is wound around the pulley and lift moves.

Rotation is a necessary movement for the changing 


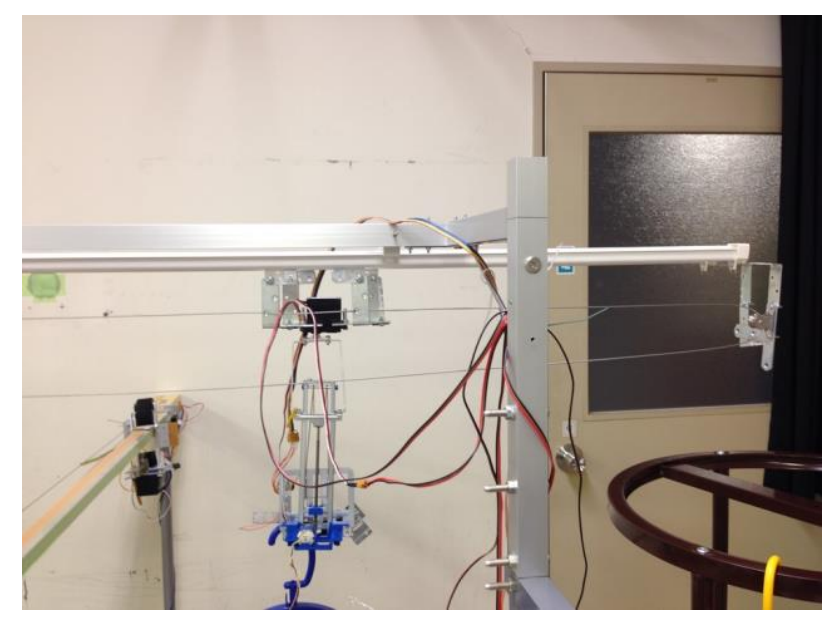

Fig. 10. Mechanism for taking out the hanger.

the orientation of the hanger. It is rotated by a servo motor attached directly to the lift top. Thus the hanger taken out of the rotary hanger rack can be hung on the single hanger rack.

\section{Experiment}

\subsection{Performance of the mechanism for taking out the hanger}

We checked the performance of the mechanism for taking out the hanger. We measured the time of the hanger transportation. Measurement was done between the sensor of the lift detect the hanger on the rotary hanger rack and the hanger was transported the single hanger rack of the robot body.

\subsection{Performance of the whole system}

We checked the performance of whole system. We confirmed whether folded up in succession a plurality of T-shirts in the entire system.

\section{Result}

\subsection{Result of the experiment about takes out the hanger system}

We checked the performance of the mechanism for taking out the hanger. As a result, the mechanism takes about 30 seconds to carry the hanger.

\subsection{Result of the experiment about whole system}

We checked the performance of whole system. As a result, it couldn't fold more than three until now, by the improvement of the hanger eject mechanism, now it can fold more T-shirts.

\section{Conclusions}

We developed a system for folding T-shirts hanging state, and has undergone improvements to fold up many T-shirt in succession. But still it is not possible to fold anything other than T-shirts in this system. Therefore, we will further improve the system, and we want to perfect it.

\section{References}

(1) KAWABATA Shinichiro : "Technological Progress of Washing Machine", Journal of Japan Society of Mechanical Engineers, Vol. 115, No. 1120, pp. 146-147, 2012

(2) NISHIWAKI S. : "Tecqnology Trend of Washing Machine", Journal of Japan Society of Mechanical Engineers, Vol. 115, No. 1120, pp. 146-147, 2012

(3) OSAWA Fumiaki, KAKIKURA Masayoshi : "A Design Method for Effective Grasping of Washed Clothes”, JRSJ, Vol. 19 . No. 6. pp. 59-67, 2001.

(4) Ryunosuke Miyamoto, Yuhki Kitazono, : "Development of Robot to Fold T-shirt in the State of Haning", The Japanese Journal of the Institute of Industrial Applications Engineers, Vol.3, No.1, pp.24-33, 2015

(5) Weikert, S., Ratnaweera, R., Zirn, O., \& Wegener, K. : "Modeling and Measurment of H-bot kinematic systems".Zurich : ETH Institute for Machine Tools and Manufacturing,2011

(6) Nanami Tajima, Koji Tsukada, Itiro Siio. : "AwareHanger:Context-aware hanger to inform users of laundry states", IPSJ SIG Technical Report, Vol. 2010-UBI-27, No. 2, pp. 1-5, 2010 